\title{
QUANTITATIVE STRUCTURE-ACTIVITY RELATIONSHIP ANALYSIS OF NOVEL PYRAZOLINE DERIVATIVES USING $K$ NEAREST NEIGHBOUR MOLECULAR FIELD ANALYSIS METHOD
}

\author{
DEEPALI M. JAGDALE, RAMAA C. . $^{*}$ \\ Department of Pharmaceutical Chemistry, Bharati Vidyapeeth's College of Pharmacy, Sector 8, C. B. D. Belapur, Navi Mumbai, India, \\ 400614 \\ Email: sinharamaa@yahoo.co.in
}

Received: 25 Apr 2017 Revised and Accepted: 02 Nov 2017

\begin{abstract}
Objective: Malonyl CoA decarboxylase (MCD) enzyme plays important role in fatty acid and glucose oxidation. Inhibition of MCD might turn to a novel approach to treat ischemia. The main objective of this research article was to develop a novel pharmacophore for enhanced activity.
\end{abstract}

Methods: Three-dimensional quantitative structure-activity relationships (3D-QSAR) was performed for pyrazoline derivatives as MCD inhibitors using VLife MDS 4.6 software. The QSAR model was developed using the stepwise 3D-QSAR $k$ NN-MFA method.

Results: The statistical results generated from $k$ NN-MFA method indicated the significance and requirements for better MCD inhibitory activity. The information rendered by 3D-QSAR model may render to better understanding and designing of novel MCD inhibitors.

Conclusion: 3D-QSAR is an important tool in understanding the structural requirements for the design of novel and potent MCD inhibitors. It can be employed to design new drug discovery.

Keywords: 3D-QSAR, MCD inhibitors, $k$ NN-MFA model, Stepwise variable selection method

(C) 2017 The Authors. Published by Innovare Academic Sciences Pvt Ltd. This is an open-access article under the CC BY license (http://creativecommons.org/licenses/by/4.0/) DOI: http://dx.doi.org/10.22159/ijpps.2017v9i12.19401

\section{INTRODUCTION}

Cardiovascular disease, being one of the leading causes of death in the world, is continuing to act as a major problem of health in both developing and developed countries. Impaired cardiac efficiency, which is one of the important contributors to cardiac disease, is caused due to inadequate oxygen supply to the heart (ischemic condition) [1-3]. After the development of ischemia, increased fatty acid level in circulation causes increased fatty acid oxidation and decreased glucose oxidation by heart cells. This leads to uncoupling of glucose oxidation and glycolysis, causing heart cells' acidosis [4-5].

Taking this into consideration, a variety of malonyl CoA decarboxylase (MCD) inhibitors have been synthesized by the researchers [6-8]. MCD carries out decarboxylation of malonyl-CoA to acetyl-CoA. Therefore, inhibition of MCD increases the levels of malonyl-CoA, which further reduces fatty acid oxidation and increases glucose oxidation in the mitochondria of heart cells. Increase in glucose oxidation has two benefits, (i) reduction in acidosis of heart cells and, (ii) increased ATP production, causing more energy supply to heart cells [9-12]. These observations affirm the further scope and need for the development of newer MCD inhibitors for ischemia treatment.

We selected Pyrazoline as a scaffold, as it is an important building block in medicinal chemistry and led to the discovery of a number of derivatives such as anticonvulsant, antitumor, antibacterial, antiviral, antidiabetic etc [13-16]. Quantitative structure-activity relationship studies done by Patel and Talele (2007) indicated that MCD inhibitors should contain a heteroaromatic ring bearing an acidic proton [17]. In view of these and as a part of our study, it was thought of interest to combine the pyrazoline ring and hydrophobic phenyl ring having phenolic acidic proton to get 3, 5-diaryl-(pyrazol1-yl)-1, 3-thiazole-4(5H)-one derivatives (derivatives 1-10, table 1 ) 3, 5-diaryl-(pyrazol-1-yl)-ethanone derivatives (derivatives 11-18, table 1) and perform 3D QSAR studies on them, which would be helpful for the researchers in future to design MCD inhibitors. Herein, we describe 3D-QSAR studies of the pyrazoline derivatives (derivatives 1-18).

\section{MATERIALS AND METHODS}

\section{Data sets and biological activity}

The dataset of 18 pyrazoline derivatives, which were synthesized by us by using previously reported method [18-19] was used for generation of 3D-QSAR model by using the molecular modeling software package VLife molecular design suite (VLife MDS) version 4.6 [20] on HP-PC (HPLV1911) with a Pentium IV processor and Windows 7 operating system. The structures of pyrazoline derivatives and their MCD inhibitory activity in the form of IC $_{50}$ values are mentioned in table 1.

\section{D-QSAR studies}

\section{Ligand preparation}

VLifeMDS main window, which provides a link for 2D drawing builder was used to draw 2D structures of pyrazoline derivatives. All the molecules were converted from 2D format to 3D format. All the 3D structures were optimized using Merck molecular force field (MMFF) with 100000 as the maximum number of cycles, 0.01 as convergence criteria, and 1.0 as the medium's dielectric constant. Analytical was selected as gradient type and non-bonded cut off was set at 20 and 10 for electrostatic and $\mathrm{vdW}$ respectively [21].

The total potential energy of a confirmation can be calculated as the

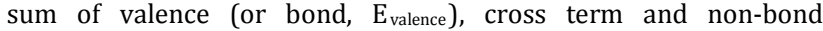
interactions ( $\left.\mathrm{E}_{\text {non bond }}\right)$.

$$
\begin{aligned}
& E_{\text {valence }}=E_{\text {bond }}+E_{\text {angle }}+E_{\text {torsion }}+E_{\text {oop }}+E_{\text {UB }} \\
& E_{\text {non bond }}=E_{\text {vdW }}+E_{\text {coulomb }}+E_{H} \text { bond }
\end{aligned}
$$

Where,

$\mathrm{E}_{\text {bond }}=$ energy of bond stretching;

Eangle = energy of valence angle bending;

$\mathrm{E}_{\text {torsion }}=$ energy of dihedral angle torsion;

$\mathrm{E}_{\text {oop }}=$ inversion energy, also called out-of-plane interactions; 
$\mathrm{E}_{\text {uв }}=\mathrm{A}$ Urey-Bradley term which accounts for interactions between atom pairs involved in 1-3 configurations (i.e. atoms bound to a common atom);

$\mathrm{E}_{\mathrm{vdW}}=$ van der Waals energy;

$\mathrm{E}_{\text {coulomb }}=$ electrostatic energy;

$\mathrm{E}_{\mathrm{H} \text { bond }}=$ energy of hydrogen bond.

\section{Alignment of molecules}

Alignment of the molecules in 3D space is an important step in 3DQSAR to obtain optimal alignment between the molecular structures necessary for ligand-receptor interaction, which is related to the conformational flexibility of molecules.

All the optimized molecules were aligned by using a template based method with the help of VLife MDS 4.6 template based alignment tool, using pyrazoline as a template, which was the common substructure in the series.

The most active compound was chosen as a reference molecule (table 1). All the other molecules in the data set were aligned on reference molecule, considering the template as a basis for alignment [22]

Table 1: Structures and biological activities of pyrazoline derivatives

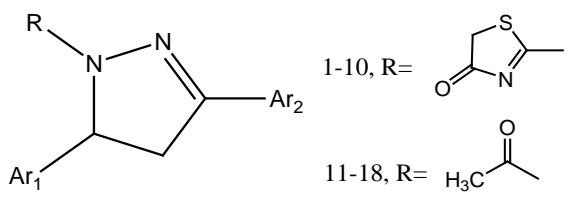

\begin{tabular}{lll}
\hline Code & Ar $_{\mathbf{1}}$ & $\mathbf{A r}_{\mathbf{2}}$ \\
\hline 1 & Phenyl & 4-hydroxyphenyl \\
2 & 4-methoxyphenyl & 4-hydroxyphenyl \\
3 & 3-thiophenyl & 4-hydroxyphenyl \\
4 & 4-bromophenyl & 4-hydroxyphenyl \\
5 & 3-furanyl & 4-hydroxyphenyl \\
6 & 2,4-dinitrophenyl & 4-hydroxyphenyl \\
7 & 2-nitro-4-methoxyphenyl & 4-hydroxyphenyl \\
8 & 3-dimethoxyphenyl & 4-hydroxyphenyl \\
9 & 4-nitrophenyl & 4-hydroxyphenyl \\
10 & 2-pyridyl & 4-hydroxyphenyl \\
11 & 2,4-dichlorophenyl & 4-hydroxyphenyl \\
12 & 2,4-difluorophenyl & 4-hydroxyphenyl \\
13 & 2,4-dibromophenyl & 4-hydroxyphenyl \\
14 & 3-thiophenyl & 4-hydroxyphenyl \\
15 & 3-furanyl & 4-hydroxyphenyl \\
16 & 2,4-dimethoxyphenyl & 4-hydroxyphenyl \\
17 & 4-chlorophenyl & 4-hydroxyphenyl \\
18 & 3,4-dichlorophenyl & 4-hydroxyphenyl \\
\hline
\end{tabular}

\section{Computation of steric, electrostatic and hydrophobic fields}

All the aligned molecules were used for calculation of molecular local shape field descriptors. Using MMFF and Del-re charge type, field descriptors such as electrostatic, steric and hydrophobic were calculated. Considering the distance-dependent dielectric function probe for carbon atom as 1.0, the dielectric constant was set as 1.0. Cut off values for electrostatic and steric were set as $10.0 \mathrm{kcal} / \mathrm{mole}$ and $30.0 \mathrm{kcal} /$ mole respectively.

This resulted in the calculation of 3120 descriptors (1040 each for, electrostatic, steric and hydrophobic). For performing a robust QSAR analysis descriptors that show variation for all the molecules are important. A descriptor that is constant for all the molecules will not contribute to QSAR and hence were removed from the worksheet. Experimental activity was selected as the dependent variable, whereas remaining descriptors were selected as independent variables [23].

\section{KNN-MFA model for 3D-QSAR}

$K$-nearest neighbour, molecular field analysis ( $k$ NN-MFA) method was developed which requires suitable alignment of a given set of molecules [24]. This method involves generation of a common rectangular grid around the molecules.

The steric and electrostatic interaction energies are computed at the lattice points of the grid using a methyl probe of charge +1 . These interaction energy values are considered for relationship generation and utilized as descriptors to decide nearness between molecules.

$k N N-M F A$ model was generated using the stepwise variable (SW) selection method. This method considers the variables stepwise for calculation. The forward method was selected wherein; procedure begins by developing a trial model with a single step at a time adding variables. This method continues until there are no more significant variables remaining outside the model.

The cross-correlation limit was set to 0.5. A number of variables were set to 4 , equalled to the number of molecules divided by 5 . A number of maximum neighbours were set at 5 whereas; the number of minimum neighbours was set at 2 .

\section{RESULTS AND DISCUSSION}

\section{D-QSAR studies}

In the present study, 3D-QSAR by a $k N N-M F A$ model coupled with a stepwise variable selection method was developed for 18 novel 3, 5diaryl-(pyrazol-1-yl)-1, 3-thiazole-4(5H)-one derivatives (1-10) and 3, 5-diaryl-(pyrazol-1-yl)-ethanone derivatives (11-18) based on steric, electrostatic and hydrophobic fields. The structures of these derivatives are shown in table 1.

An energetically stable and highly bioactive compound was chosen as a reference molecule. On this reference molecule, other molecules in the data set were aligned, considering template as the basis for the alignment. The training set and test set were selected on random selection method. Compounds 2, 5, 9, 15 got selected as the test set and remaining 14 compounds got selected as the training set (table 2). Predicted biological activities of all compounds in the test set were found to lie within the minimum and maximum value range of biological activities of the training set of compounds (fig. 1).

This proved the correctness of the selection of the training set and test set. 


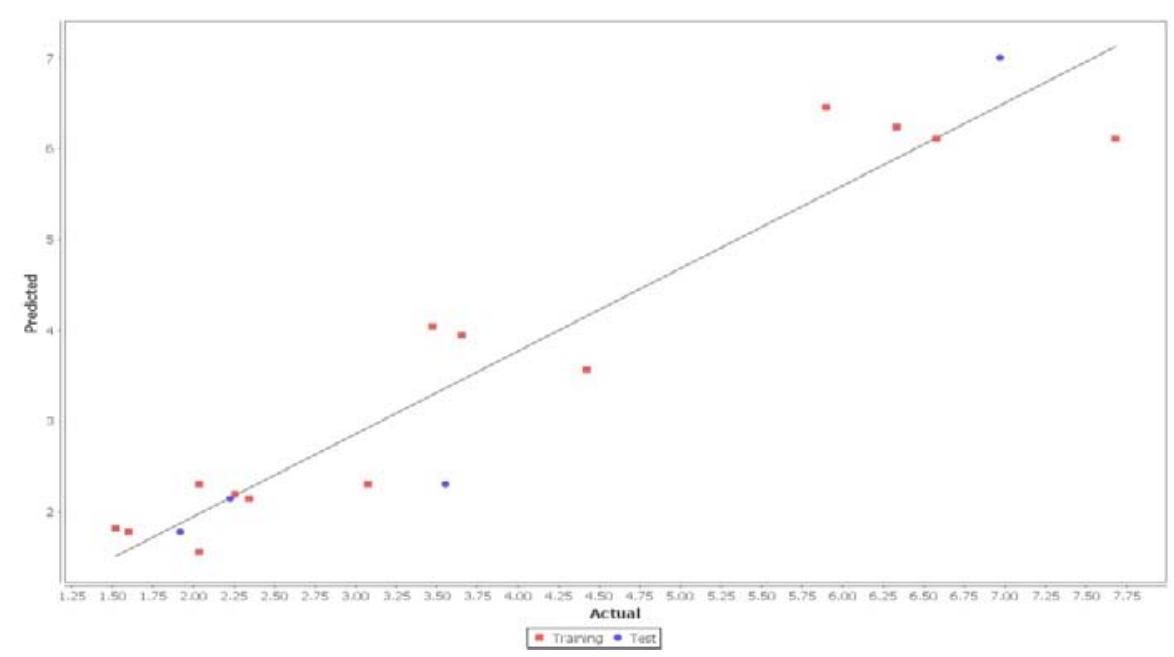

Fig. 1: Comparison of observed activity versus predicted activity for the training set and test set according to 3D-QSAR model by SW-kNNMFA method

Table 2: Observed and predicted activity along with residual by QSAR equation

\begin{tabular}{|c|c|c|c|c|}
\hline \multirow[t]{2}{*}{ Code } & \multirow[t]{2}{*}{ Sets } & \multicolumn{2}{|l|}{$\mathrm{IC}_{50} \mu \mathrm{M}$} & \multirow[t]{2}{*}{ Residual } \\
\hline & & Experimental & Predicted & \\
\hline 1 & training & 2.03 & 1.56 & 0.47 \\
\hline 2 & test & 3.55 & 2.3 & 1.25 \\
\hline 3 & training & 1.6 & 1.78 & -0.18 \\
\hline 4 & training & 1.52 & 1.82 & -0.3 \\
\hline 5 & test & 2.23 & 2.15 & 0.08 \\
\hline 6 & training & 2.04 & 2.3 & -0.26 \\
\hline 7 & training & 2.26 & 2.19 & 0.07 \\
\hline 8 & training & 3.08 & 2.3 & 0.78 \\
\hline 9 & test & 1.92 & 1.78 & 0.14 \\
\hline 10 & training & 2.34 & 2.15 & 0.19 \\
\hline 11 & training & 6.58 & 6.11 & 0.47 \\
\hline 12 & training & 3.65 & 3.95 & -0.3 \\
\hline 13 & training & 5.9 & 6.45 & -0.55 \\
\hline 14 & training & 6.33 & 6.24 & 0.09 \\
\hline 15 & test & 6.97 & 7.01 & -0.04 \\
\hline 16 & training & 7.68 & 6.11 & 1.568 \\
\hline 17 & training & 4.42 & 3.56 & 0.86 \\
\hline 18 & training & 3.47 & 4.04 & -0.568 \\
\hline
\end{tabular}

From table 2, it is evident that predicted activities of all the compounds are in good agreement with their corresponding experimental activities and the optimal fit is obtained. The plot of predicted versus observed activities of both training and test set compounds (fig. 1) helped in cross-validation of $k$ NN-MFA QSAR model. Various statistically significant models were generated using stepwise variable selection method, of which the best model having pred $\mathrm{r}^{2}=0.9008$ and $\mathrm{q}^{2}=0.9061$ is discussed herein (table 3 ).

Table 3: Statistical results of 3D-QSAR $k$ NN-MFA model generated by stepwise variable selection method

\begin{tabular}{lll}
\hline S. No. & Statistical parameter & Results \\
\hline 1 & n (Training/Test) & $14 / 4$ \\
2 & $k$ nearest neighbour & 2 \\
3 & $\mathrm{n} \quad 14$ & 11 \\
4 & Degree of freedom & 0.9061 \\
5 & $\mathrm{q}^{2}$ & 0.6327 \\
6 & $\mathrm{q}^{2}$ se & 0.9008 \\
7 & Pred_r & 0.7292 \\
8 & Pred_r ${ }^{2}$ se & S_304-0.5101-0.4930 \\
9 & Descriptors (range) & S_305 0.38170 .3830 \\
& & \\
\hline
\end{tabular}

From statistical results generated by stepwise variable selection 3DQSAR $k$ NN-MFA (SW- $k$ NN-MFA) model (table 3, fig. 2), the points generated are S_304 (-0.5101-0.4930) and S_305 (0.3817 0.3830) i.e. the steric interaction between the probe $\left(\mathrm{CH}_{3}\right)$ at their corresponding spatial grid points of 304 and 305. These points suggested the significance and requirement of steric properties for a better MCD inhibitory activity. The negative range at grid point s_304 on thaizolone moiety indicates that negative steric potential is 
favorable for an increase in MCD inhibitory activity of pyrazoline derivatives and hence less bulky substituent is preferred in that region. Whereas, the positive range at grid point s_305 on acetyl moiety indicates that positive steric potential is favourable for an increase in biological activity and hence bulky group is favourable for an increase in the activity.

Hence, intermediate steric group's like- $\mathrm{COCH}_{2} \mathrm{CH}_{3},-\mathrm{CONH}_{2}$,$\mathrm{CONHNH}_{2},-\mathrm{CSNH}_{2},-\mathrm{CSNHCH}_{3}$ etc. are preferred on 1-position of pyrazoline ring. These results are in close agreement with the experimental observations that, compounds 11-18 with thiazolone moiety showed more MCD inhibitory activity than acetyl substituted 1-10 compounds. Further, these findings are also in compliance with results obtained from QSAR studies done by Patel MR et al. [17]. In their study, they found that structural variation of substituents present on ureide nitrogen plays important role in the MCD inhibitory activity.

Studies done by Patel MR et al. indicated that hydroxy group present in 1,1,1,3,3,3-hexafluro-2-propanol derivatives is hydrogen bond acceptor and is important for activity.

Whereas, in our study spatial grid point is not generated at the phenolic hydroxy group. The absence of spatial grid point doesnot necessarily imply that phenolic hydroxy group does not contribute to MCD inhibitory activity but, rather all molecules contain same functionality.

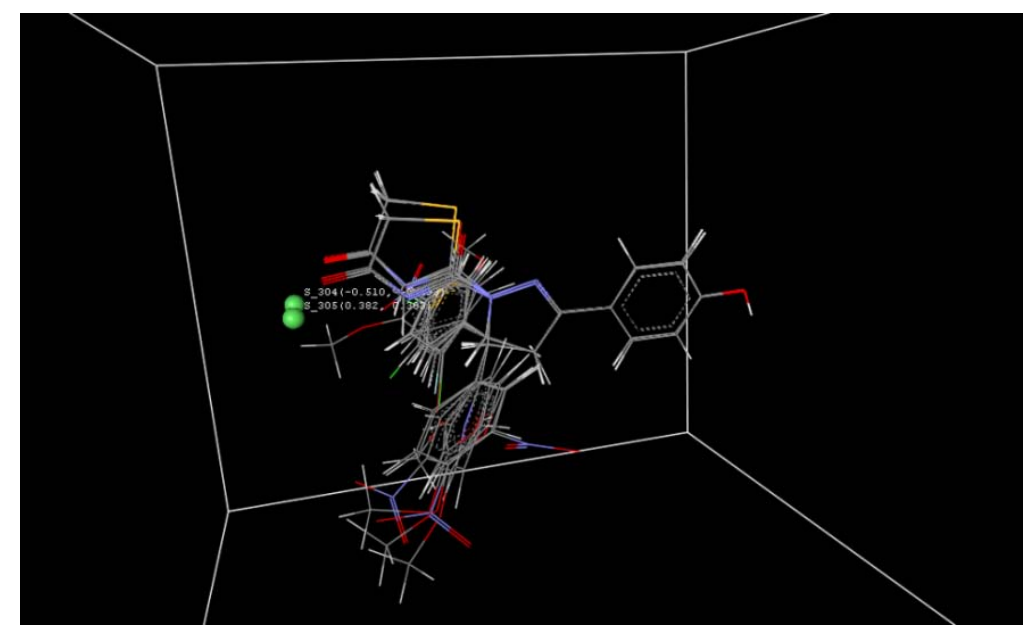

Fig. 2: Stereo view of the molecular rectangular grid around superimposed molecular units of pyrazoline derivatives using SW-kNN-MFA method

Thus, the contribution plot arising out of 3D-QSAR studies provides useful insights for better understanding of the structural features of pyrazoline derivatives responsible for producing significant MCD inhibitory activity.

\section{CONCLUSION}

Statistically significant 3D-QSAR $k$ NN-MFA model having pred $\mathrm{r}^{2}=$ 0.9008 and $\mathrm{q}^{2}=0.9061$ was generated by using a stepwise variable selection method for a series of pyrazoline derivatives using VLifeMDS 4.6 software. On the basis of the correlation coefficient, this model was accepted for 3D QSAR study [25]. The contribution plot of steric, electrostatic and hydrophobic field interactions generated by $3 \mathrm{D}-\mathrm{QSAR}$ revealed that intermediate bulky groups such as- $\mathrm{COCH}_{2} \mathrm{CH}_{3},-\mathrm{CONH}_{2}$,- $-\mathrm{CONHNH}_{2}$,- $-\mathrm{CSNH}_{2}$,- $-\mathrm{CSNHCH}_{3}$ etc. are favourable at 1-position of pyrazoline ring. This finding is in close agreement with the structures of these compounds, where less biological activity is observed in compounds 11-18 with an acetyl group and more biological activity is observed in compounds 1-10 with thiazolone group. In conclusion, the model provided by this work can be employed to design new derivatives of pyrazoline with increased MCD inhibitory activity.

\section{ACKNOWLEDGEMENT}

The authors would like to thank to an institution for providing all facilities to carry out work and VLife technology Pvt. Ltd. for their guidance and training in carrying out 3D QSAR study.

\section{AUTHORS CONTRIBUTION}

Dr. C. S. Ramaa is the principal author and guided the work.

Mrs. Deepali Jagdale was responsible for 3D QSAR analysis and manuscript writing.

\section{CONFLICTS OF INTERESTS}

The authors confirm that this article content has no conflicts of interest.

\section{REFERENCES}

1. Ussher JR, Lopaschuk GD. The malonyl-CoA axis as a potential target for treating ischemic heart disease. Cardiovasc Res 2008;79:259-68.

2. Stanley WC, Recchia FA, Lopaschuk GD. Myocardial substrate metabolism in the normal and failing heart. Physiol Rev 2005;85:1093-129.

3. Frink RJ. Sudden cardiac death is not caused by ischemia. Int J Cardiovasc Res 2012;1:1-2.

4. Jaswal JS, Keung W, Wang W, Ussher JR, Lopaschuk GD. Targeting fatty acid and carbohydrate oxidation-A novel therapeutic intervention in the ischemic and failing heart. Biochim Biophys Acta 2011;1813:1333-50.

5. Fillimore N, Lopaschuk GD. Targeting mitochondrial oxidative metabolism as an approach to treat heart failure. Biochim Biophys Acta 2013;1833:857-65.

6. Dyck JRB, Cheng JF, Stanley WC, Rick B, Chandler MP, Brown S, et al. Malonyl coenzyme A decarboxylase inhibition protects the ischemic heart by inhibiting fatty acid oxidation and stimulating glucose oxidation. Circ Res 2004;94:e78-e84.

7. Bandopadhyay GK, Yu JG, Ofrecio J, Olefsky JM. Increased malonyl CoA levels in muscle from obese and type 2 diabetic subjects lead to decreased fatty acid oxidation and increased lipogenesis; Thiazolidinedione treatment reverses these defects. Diabetes 2006;55:2277-85.

8. Walters AM. Mitochondria as a drug target in ischemic heart disease and cardiomyopathy. Circ Res 2012;111:1222-36.

9. Dyck JRB, Lopaschuk GD. Malonyl-CoA control of fatty acid oxidation in the ischemic heart. J Mol Cell Cardiol 2002;34:1099-109.

10. Cheng JF, Liu B, Angeles L, Arrhenius T, Mar D, Wilson ME, et al. Cyanoguanidine based azole compounds useful as malonyl-CoA decarboxylase inhibitors. U. S. Patent 2005;7:786, 145.

11. Clifford DL, Lopaschuk GD. Role of malonyl-CoA in heart disease and the hypothalamic control of obesity. Cardiovasc Res 2007;73:278-87. 
12. Abel ED. Glucose for the aging heart. Circ 2007;116:884-7.

13. Havrylyuk D, Zimenkovsky B, Vasylenko O, Zaprutko L, Gzella A, Lesky R. Synthesis of novel thiazolone based compounds containing pyrazoline moiety and evaluation of their anticancer activity. Eur J Med Chem 2009;44:1396-404.

14. Shaharyar M, Siddiqui AA, Ali MA, Sriram D, Yogeeswari P. Synthesis and in vitro antimycobacterial activity of N1nicotinoyl-3-(4'-Hydroxy-3'-Methyl Phenyl)-5 [(sub) phenyl] 2Pyrazolines. Bioorg Med Chem Lett 2006;16:3947-9.

15. Dipankar B, Hirakmoy C, Asish B, Abhijit C. 2-pyrazoline: a pharmacologically active moiety. Int Res J Pharm Appl Sci 2011;1:68-80.

16. Abdel-Aziz M, Gamal-Eldeen AM. Synthesis and screening of anti-cancer, antioxidant, and anti-inflammatory activities of novel galloyl pyrazoline derivatives. Pharm Biol 2009;47: 854-63.

17. Patel MR, Talele TT. 3D-QSAR studies on malonyl coenzyme A decarboxylase inhibitors. Bioorg Med Chem 2007;15:4470-80.

18. Gandhi PS, Gaikwad PL, Jagdale DM, Kadam VJ. Synthesis, characterization and antimicrobial studies of novel 2pyrazoline derivatives containing thiazolone moiety. Int J Univ Pharm Biosci 2013;2:648-58.

19. Jagdale DM, Ramaa CS. Design, synthesis and in vitro evaluation of some small molecules malonyl CoA decarboxylase inhibitors containing pyrazoline scaffold and study of their binding interactions with malonyl CoA decarboxylase via preliminary docking simulation. Med Chem Res 2017;26:2127-40.

20. VLife Molecular Design Suite 4.6, V Life Sciences Technologies Pvt. Ltd; www.vifescience.com. [Last accessed on 20 Mar 2017]

21. Ajmani S, Jadhav K, Kulkarni S. Three dimensional QSAR using the k-Nearest Neighbour Method and its interpretation. J Chem Inf Model 2006;46:24-31.

22. Balaje R, Dhanarajan MS. 3D QSAR studies of identified compounds as potential inhibitors for anti-hyperglycemic targets. Asian J Pharm Clin Res 2014;7:362-4.

23. Shen M, LeTiran A, Xiao Y, Golbraikh A, Kohn H, Tropsha A. Quantitative structure-activity relationship analysis of functionalized amino acid anticonvulsant agents using $k$ Nearest neighbor and simulated annealing PLS methods. J Med Chem 2002;45:2811-23.

24. Jain SV, Ghate M, Bhandoria KS, Bari SB, Chaudhary A, Borse JS. 2D, 3D-QSAR and docking studies of 1,2,3-thiadiazole thioacetamides analogues as potent HIV-1 non-nucleoside reverse transcriptase inhibitors. Org Med Chem Lett 2012;2:1-13.

25. Raji K, Muthukumar V. Quantitative structure-activity relationship analysis of the anticonvulsant activity of erythrinine. Asian J Pharm Clin Res 2016;9:125-9. 